\title{
Article \\ Clinical Efficacy and Safety of Low-Level Laser Therapy in Patients with Perennial Allergic Rhinitis: A Randomized, Double-Blind, Placebo-Controlled Trial
}

\author{
Hahn Jin Jung ${ }^{1}\left(\mathbb{D}\right.$, , Young-Jun Chung ${ }^{2,3,4}$, Young-Seok Choi ${ }^{1}$, Phil Sang Chung ${ }^{2,3,4}$ and Ji-Hun Mo ${ }^{2,3,4, *}$ \\ 1 Department of Otorhinolaryngology-Head and Neck Surgery, Chungbuk National University College \\ of Medicine, Chungbuk National University Hospital, Cheongju 361711, Korea; hahnjin2@naver.com (H.J.J.); \\ yschoi@chungbuk.ac.kr (Y.-S.C.) \\ 2 Department of Otorhinolaryngology, Dankook University College of Medicine, Cheonan 31116, Korea; \\ entdocjung@daum.net (Y.-J.C.); pschung@dankook.ac.kr (P.S.C.) \\ 3 Beckman Laser Institute Korea, Dankook University College of Medicine, Cheonan 31116, Korea \\ 4 Laser Translational Clinical Trial Center, Dankook University Hospital, Cheonan 31116, Korea \\ * Correspondence: jihunmo@gmail.com; Tel.: +82-40-550-3933
}

check for

updates

Citation: Jung, H.J.; Chung, Y.-J.; Choi, Y.-S.; Chung, P.S.; Mo, J.-H. Clinical Efficacy and Safety of Low-Level Laser Therapy in Patients with Perennial Allergic Rhinitis: A Randomized, Double-Blind, Placebo-Controlled Trial. J. Clin. Med. 2021, 10, 772. https://doi.org/ $10.3390 /$ jcm10040772

Academic Editor: Alfonso Del Cuvillo

Received: 18 January 2021

Accepted: 11 February 2021

Published: 15 February 2021

Publisher's Note: MDPI stays neutral with regard to jurisdictional claims in published maps and institutional affiliations.

Copyright: (c) 2021 by the authors. Licensee MDPI, Basel, Switzerland. This article is an open access article distributed under the terms and conditions of the Creative Commons Attribution (CC BY) license (https:// creativecommons.org/licenses/by/ $4.0 /)$.

\begin{abstract}
Allergic rhinitis (AR) is a common disease that interferes with the daily activities and reduces the quality of life. Conventional treatments often do not provide complete resolution of the symptoms, and many new treatment modalities have been tried. This study aimed to evaluate the efficacy and safety of low-level laser therapy (LLLT) for AR in a randomized, double-blind, placebocontrolled trial. Patients diagnosed with AR were randomly allocated to receive LLLT or sham treatment. The primary outcome was a change in the reflective total nasal symptom score (TNSS). The secondary outcome was quality of life scores assessed using the Rhinoconjunctivitis Quality of Life Questionnaire. Incidences of adverse events were also recorded. Among 67 randomized subjects, 41 subjects (22 in LLLT group and 19 in sham treatment group) were included for efficacy analysis. The LLLT group showed a significantly improved TNSS score compared to the sham treatment group for decreasing AR symptom severity $(p=0.011)$ and improving quality of life regarding nasal symptoms $(p=0.036)$ at the end of treatment. Throughout the treatment period, no severe adverse events occurred. This clinical trial showed that LLLT is an effective and safe option for the management of AR regarding symptom relief and quality of life improvement.
\end{abstract}

Keywords: allergic rhinitis; low-level laser therapy; randomized controlled trial; quality of life; treatment

\section{Introduction}

Allergic rhinitis (AR) is a chronic airway disorder and one of the most common health problems [1]. AR is associated with a substantial health and psychological burden to patients due to its high incidence, etiologically complex, and prolonged disease course [2,3]. $\mathrm{AR}$ is characterized by nasal symptoms such as congestion, sneezing, rhinorrhea, and itching [4]. Although these symptoms are usually considered nonserious, they do affect the daily and social activities of an individual; they sometimes have significant effects on the quality of life and even result in considerable medical expenses $[5,6]$. In addition, the prevalence of perennial AR has been increasing in recent years [7].

The standard treatment for AR includes patient education, avoidance of allergen, medical treatment, and immunotherapy. However, these treatments frequently do not provide complete resolution of the symptoms. Adverse effects of the medications include drowsiness and dryness of the throat, which can disturb the patient's quality of life almost as much as the symptoms they are trying to alleviate [8].

Among several trials regarding the treatment of AR, low-level laser therapy (LLLT) has been reported to reduce the symptoms of AR and improve the quality of life [9-15]. 
Furthermore, evidence of its anti-inflammatory and immunosuppressive effects has also been reported $[16,17]$. LLLT does not only improve AR symptoms, but also decreases nasal mucosal interleukin (IL)-5 levels and reduces the eosinophil count [10]. In an animal study, a low-level laser $(658 \mathrm{~nm}, 30 \mathrm{~mW})$ was irradiated into the intranasal cavity of an ovalbumininduced AR mouse model. It proved effective in inhibiting total immunoglobulin $\mathrm{E}$ and IL-4, and it was confirmed by histological studies to have reduced the number of infiltrated inflammatory cells in the intranasal epithelium [18]. However, previous studies had limitations in studying only moderate to severe AR patients, and allowing rescue medicine that could make interpretation of the results difficult. Therefore, there was a need for a study conducted with a larger sample under a more rigorous protocol.

In this study, we tried to clarify the efficacy and safety of LLLT in a randomized double-blind placebo-controlled trial among Korean patients with perennial AR.

\section{Materials and Methods}

\subsection{Setting}

A randomized, double-blind, and placebo-controlled study was designed to evaluate the clinical efficacy and safety of LLLT for AR treatment. The study was conducted at the Otorhinolaryngology Department of the Dankook University Hospital, Korea. The trial period was from April 2017 through March 2018. The study protocol was approved by the Institutional Review Board of Dankook University Hospital (IRB no. 2016-09-011) and was registered in the clinical research information system of South Korea (CRIS Registration Number KCT0003291). The study was conducted in accordance with the principles of the Declaration of Helsinki.

\subsection{Sample Size Calculation}

We established a sample size for an independent $t$-test using the adjusted standard deviation and the difference in total nasal symptom score (TNSS) change between the LLLT and the sham-controlled group. The effect size estimate was calculated using the results of our previous pilot study with a sample size of 40 (not published). The TNSSs in the LLLT and sham-controlled group were 2.462 and 0.786 , respectively, and the difference in TNSS change between two groups was 1.676. The standard deviation of the pilot study was adjusted for better application to the true population. With participants recruited in a 1:1 ratio in the two groups, the sample size required to yield $80 \%$ power with a twotailed significance level of $5 \%$ was calculated as 31 participants per group. To evaluate the treatment effect at the end of treatment and consider dropouts, the potential recruits were increased to up to 34 participants in each group.

\subsection{Participants}

Participants suffering from perennial AR were recruited. The inclusion criteria were: (1) participants aged older than 19 years; (2) participants suffering from mild to moderate symptoms at week 0 (baseline TNSS $\leq$ ) of AR (nasal congestion, rhinorrhea, sneezing, and nasal itching) and a proven positive allergy test (skin prick test or multiple allergen simultaneous test) to any of the common perennial allergens such as dust mites, molds, insects, and animal danders within the last 12 months; (3) participants with any gender or ethnicity; and (4) participants who signed written informed consent before they were assigned. The exclusion criteria were as follows: (1) patients younger than 18 years of age; (2) pregnant or breastfeeding women; (3) patients with definite deviated nasal septum, or sinusitis or history of operation within the last 6 months; (4) patients with hypertension, diabetes mellitus, malignancy, anemia, active pulmonary tuberculosis, infection, active respiratory disease like asthma, or other systemic diseases; (5) patients with long-term use of corticosteroids or immunosuppressive agents; (6) patients with hypersensitivity to laser or a wound at the laser site; (7) patients who were involved in another clinical study within 30 days; (8) patients who were unable to comply with the follow-up schedules; and (9) patients who had used antihistamines within 1 week, topical corticosteroids within 
2 weeks, systemic corticosteroids within 4 weeks, anti-cholinergic drugs within 3 days, antileukotriene drugs within 1 week, decongestants within 3 days, tricyclic antidepressants or phenothiazines within 2 weeks, non-steroidal analgesics within 2 weeks, and other drugs which the researchers believed were inappropriate.

\subsection{Randomization and Blinding}

Random numbers were generated by an independent statistician using a computerbased random assignment procedure. Using these random numbers, a LLLT device or sham treatment device was distributed to each participant. The sham treatment device was visually identical to the LLLT device, so neither the participants nor the physicians were aware of the list of participants in the LLLT and sham treatment groups. All participants were informed that there was a 50\% chance of receiving the LLLT or sham treatment. In addition, trial data were analyzed by a statistician who was blinded to the participants' allocation.

\subsection{Intervention}

After screening, participants who had taken the medication had washout periods and the duration of washout periods was up to 1 month, which was determined by the type of the medication. During washout period, the participants stopped taking medication. At the end of the run-in period, participants were randomized into either the LLLT group or sham treatment group by a computer-generated random number allocation scheme. The participants were treated using LLLT (multi-wavelength; AlGaInP $670 \mathrm{~nm}, 3 \mathrm{~mW}$, GaAs $830 \mathrm{~nm}, 20 \mathrm{~mW}$; Optowell Co. Ltd., Jeonju, Korea) or sham treatment for 20 min twice a day (morning and evening) for 4 weeks (Figure 1, Table 1). The parameters used for laser irradiation in the present study are shown in Table 2. The LLLT and sham treatment device was identical in shape with different parameters and has a memory that can check the usage time. Compliance was assessed based on device usage log data and usage records in the distributed diary of each participant. In this study, the participants were required to cease any medication for AR and were instructed not to use any relief medication. The intervention was terminated in cases of severe adverse events, voluntary withdrawal of participants from the study, and unpermitted medication use.
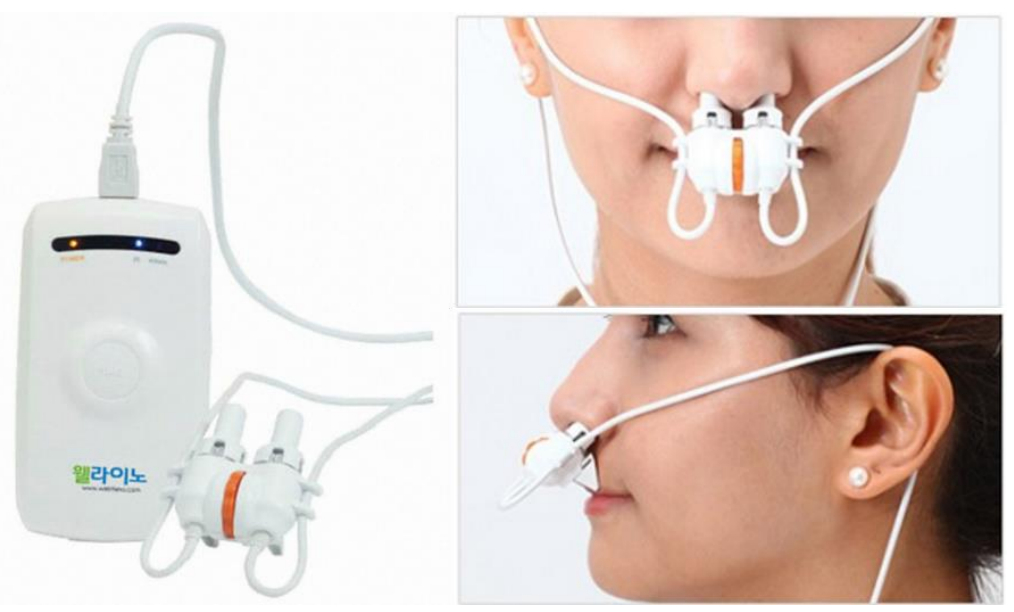

Figure 1. Device for the intranasal laser irradiation (Optowell ${ }^{\circledR}$, Jeonju, Korea). The device was applied intranasally for laser irradiation.

Table 1. Laser specifications used in low-level laser therapy.

\begin{tabular}{ccc}
\hline Laser Wavelength & Output Power & Gain Medium \\
\hline $670 \mathrm{~nm}$ (visible ray) & $670 \mathrm{~nm}$ (visible ray): $3 \mathrm{~mW}(1 \mathrm{~mW}, 3$ each) & $670 \mathrm{~nm}: \mathrm{AlGaInP}$ \\
$830 \mathrm{~nm}$ (infrared ray) & $830 \mathrm{~nm}$ (infrared ray): $20 \mathrm{~mW}$ & $830 \mathrm{~nm}: \mathrm{GaAs}$ \\
\hline
\end{tabular}


Table 2. Parameters of the irradiation device.

\begin{tabular}{cc}
\hline Product & Wellrhino \\
\hline Light source & VCSEL \\
Wavelength & 670 and $830 \mathrm{~nm}$ \\
Laser power & $670 \mathrm{~nm}, 3 \mathrm{~mW} ; 830 \mathrm{~nm}, 20 \mathrm{~mW}$ \\
Total power & $23 \mathrm{~mW}$ \\
Using Time & 20 and $40 \mathrm{~min}$ \\
\hline
\end{tabular}

\subsection{Primary Outcome}

The primary outcome was the change in TNSS [19] from baseline to the end of treatment. The primary efficacy indicators included four common symptoms of AR: nasal congestion, rhinorrhea, sneezing, and nasal itching. Each symptom was scored from 0 to 4 according to the severity ( 0 , no symptoms; 1 , mild symptoms, such as symptoms that were present but not particularly bothersome; 2 , moderate symptoms, such as symptoms that were bothersome but did not interfere with daily activities; 3 , severe symptoms such as symptoms that were bothersome and interfered with daily activities or disturbed sleep; 4 , very severe symptoms) over the previous $12 \mathrm{~h}$ time interval. The TNSS was calculated by summing the score for each of the symptoms. This questionnaire was completed by the participants at week 0 (baseline) and 4 .

\subsection{Secondary Outcome}

The secondary outcome indicators included the quality of life scored according to the Rhinoconjunctivitis Quality of Life Questionnaire (RQLQ) [20]. The RQLQ is a selfreported questionnaire which is categorized into seven areas with 28 questions regarding daily activities, sleeping state, practical problems, nasal symptoms, eye symptoms, nonnasal/eye symptoms, and emotional function domains. The participants were asked to recall their experiences during the preceding week and to rate each answer using a 7-point scale $(0$, no impairment; 6 , severe impairment). This questionnaire was completed by participants at week 0 (baseline) and week 4 .

\subsection{Adverse Events}

At each visit, the participants reported adverse events, including the specific symptom, onset, severity, duration, time of resolution, and possible association with treatment. Possible adverse events were nasal dryness, epistaxis, burning, inflammation, and nasal septum perforation. All adverse effects were analyzed at the study endpoint, regardless of whether they were considered relevant to the treatment or not. Details of the participants who withdrew due to serious adverse events were reported according to the reporting requirements.

\subsection{Statistical Analysis}

The statistical analysis was performed by a statistician blinded to the group allocation. Data were summarized as frequencies for categorical variables and means and standard deviations for continuous variables. Baseline demographic characteristics, such as sex and family history, were analyzed using the chi-squared test or an independent-samples $t$-test as appropriate.

Per-protocol population was defined as a subset of an intention-to-treat (ITT) population by excluding two subjects who took unpermitted medications during the trial. In addition, subjects whose medical device compliance was $<50 \%$ were excluded from the per-protocol set, and this population was defined as the modified per-protocol set. Efficacy analysis was performed using the modified per-protocol (mPP) set, and safety was analyzed using the per-protocol set. 
For primary outcome measures, analysis of covariance with the value before administration (ANCOVA) was performed using modified per-protocol set, and the least square means (LSMeans) of changes in each TNSS variation from baseline, adjusted for bias before treatment, were determined for each treatment group. Then, intergroup differences in LSMeans and $95 \%$ CIs during the observation period were calculated. For the primary end point, a pairwise test (between-group comparison) was performed using the above ANCOVA model and the PROC MIXED procedure in Statistical Analysis System (SAS; version 9.2, SAS Institute, Cary, NC, USA) to verify the superiority of LLLT over sham treatment. Values of $p<0.05$ were considered statistically significant. For secondary outcome measures, the two-samples independent $t$-test or the Wilcoxon rank-sum test was used to compare continuous variables as appropriate, and the chi-squared test was used to compare categorical variables between the two groups.

\section{Results}

\subsection{Participants and Baseline Characteristics}

Eighty participants were screened via enquiry. Thirteen participants who did not meet the inclusion criteria were excluded; therefore, 67 participants (ITT set) were included in the trial and were randomized to the LLLT group $(n=34)$ or sham treatment group $(n=33)$. No significant differences were observed between the groups regarding sex, underlying health status, and laboratory test results. However, there was a significant difference in the mean age between the two groups ( $27.82 \pm 6.74$ years for the LLLT group and $33.79 \pm 12.28$ years for the sham treatment group, $p=0.018)$. There were no significant differences in TNSS or RQLQ between the two groups at baseline ( $p>0.05$, Table 3). During the four week treatment period, two participants in the LLLT group discontinued due to unpermitted medication use; therefore, of the 67 participants in the ITT group, 65 participants (perprotocol (PP)) were defined. Among the 65 patients in the PP set, 10 in the LLLT group and 14 in the sham treatment group had less than $50 \%$ compliance. Excluding these participants with lower compliance, a total of 41 participants (22 in the LLLT group and 19 in the sham treatment group) were categorized as the modified per-protocol (mPP) set (Figure 2).

Table 3. Comparison of participant characteristics in the two groups (intention-to-treat).

\begin{tabular}{cccc}
\hline Variable & $\begin{array}{c}\text { LLLT } \\
(\boldsymbol{n}=\mathbf{3 4})\end{array}$ & $\begin{array}{c}\text { Sham Treatment } \\
(\boldsymbol{n}=\mathbf{3 3})\end{array}$ & $\boldsymbol{p}$-Value \\
\hline Age (years) & $27.8 \pm 6.7$ & $33.8 \pm 12.3$ & 0.018 \\
Gender (male:female) & $17: 17$ & $13: 20$ & 0.383 \\
TNSS & $5.94 \pm 3.29$ & $6.64 \pm 2.06$ & 0.243 \\
RQLQ & $58.29 \pm 26.06$ & $61.39 \pm 22.91$ & 0.433 \\
Daily activities & $7.00 \pm 2.76$ & $7.97 \pm 3.12$ & 0.066 \\
Sleeping state & $6.53 \pm 4.42$ & $6.00 \pm 3.30$ & 0.935 \\
Eye symptoms & $7.18 \pm 4.98$ & $7.55 \pm 5.16$ & 0.748 \\
Non-nasal/eye symptoms & $13.65 \pm 7.55$ & $13.79 \pm 6.27$ & 0.665 \\
Practical problems & $7.76 \pm 3.64$ & $8.73 \pm 3.77$ & 0.304 \\
Emotional function & $5.53 \pm 4.30$ & $6.55 \pm 3.76$ & 0.174 \\
Nasal symptoms & $10.65 \pm 4.66$ & $10.82 \pm 3.90$ & 0.823 \\
\hline
\end{tabular}

Abbreviations: LLLT, low-level laser therapy; TNSS, total nasal symptom score; RQLQ, Rhinoconjunctivitis Quality of Life Questionnaire. Values are expressed as mean \pm standard deviation. The $p$-values were obtained from a chi-squared test and Wilcoxon's rank-sum test. 


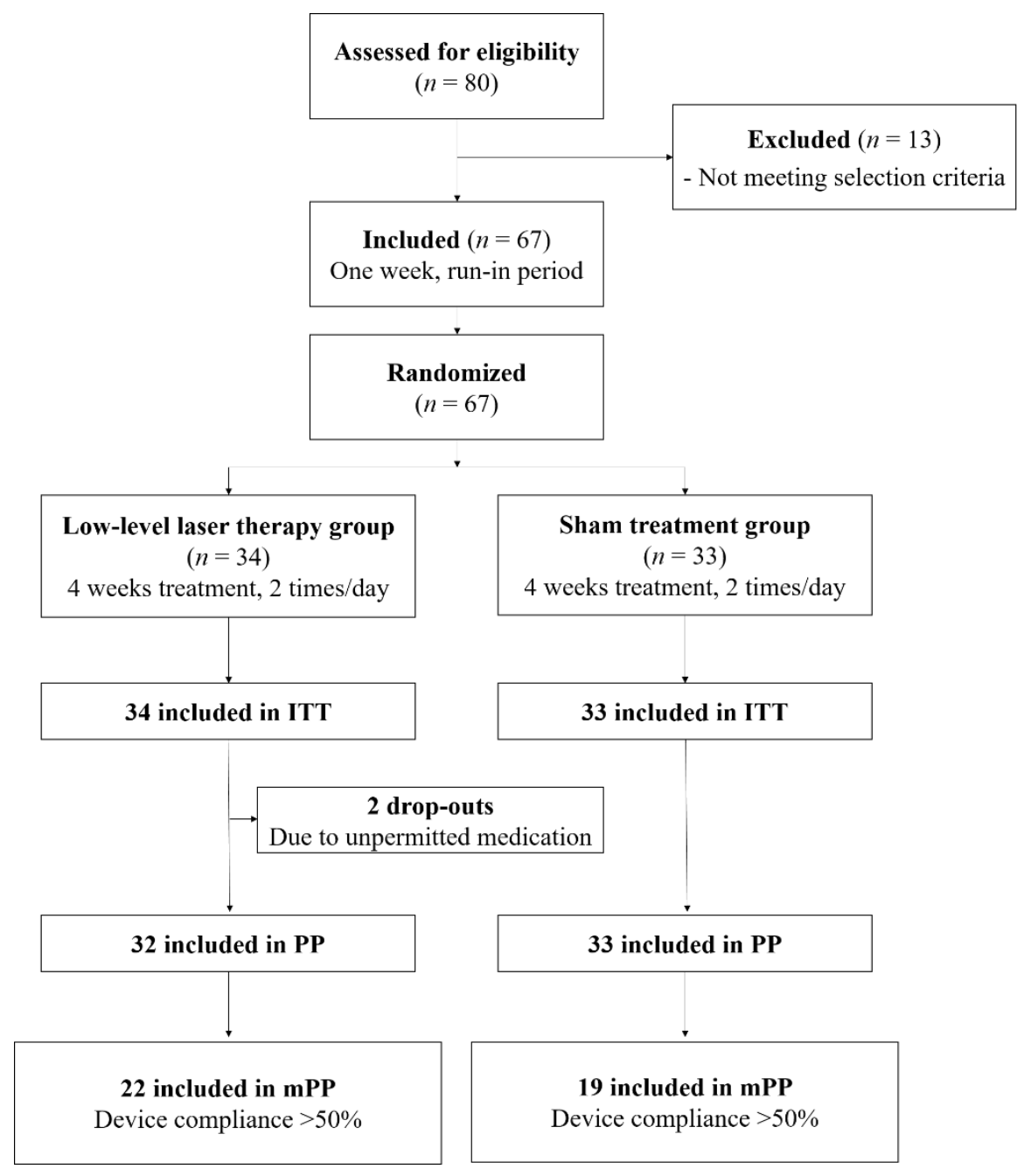

Figure 2. Flow diagram of clinical trial and disposition of patients. Abbreviations: ITT, intent to treat set; PP, per-protocol set; $\mathrm{mPP}$, modified per-protocol set.

\subsection{Primary Outcome}

$\mathrm{mPP}$ analysis showed that both the LLLT and sham treatment groups had significantly reduced TNSSs at the end of the treatment period compared with the baseline $(7.05 \pm 3.11$ to $3.32 \pm 2.85$ in the LLLT group, $p<0.001$; and $6.21 \pm 2.15$ to $4.74 \pm 2.35$ in the sham treatment group, $p=0.009$, Table 4 ). There was a greater reduction in TNSS in the LLLT group than in the sham treatment group ( $p=0.011$, Table 4$)$. The primary end point was a change (LSMeans \pm SE) in the TNSS from baseline to the end of treatment, the values for which were $-3.72 \pm 0.64$ and $-1.47 \pm 0.50$ in the LLLT and sham treatment groups, respectively (Figure 3). The between-group difference ((LLLT group)-(sham treatment group)) was -2.25 (95\% CI: -3.948 to $-0.559, p=0.011)$, which validated the hypothesis that LLLT was superior to a placebo in terms of efficacy.

Table 4. Treatment effects (TNSS, mPP set).

\begin{tabular}{ccccc}
\hline Group & Baseline & End of Treatment & Difference & $\begin{array}{c}\text { Difference } \\
\text { between Groups: } \\
\boldsymbol{p} \text {-Value }\end{array}$ \\
\hline LLLT $(n=22)$ & $7.05 \pm 3.11$ & $3.32 \pm 2.85$ & $-3.73 \pm 3.03$ & 0.011 \\
Sham treatment $(n=19)$ & $6.21 \pm 2.15$ & $4.74 \pm 2.35$ & $-1.47 \pm 2.20$ & 0.015 \\
\hline
\end{tabular}

Abbreviations: TNSS, total nasal symptom score; LLLT, low-level laser therapy; mPP set, modified per-protocol set. Values are expressed as mean \pm standard deviation. The $p$-values were obtained from a chi-squared test and Wilcoxon's rank-sum test. 


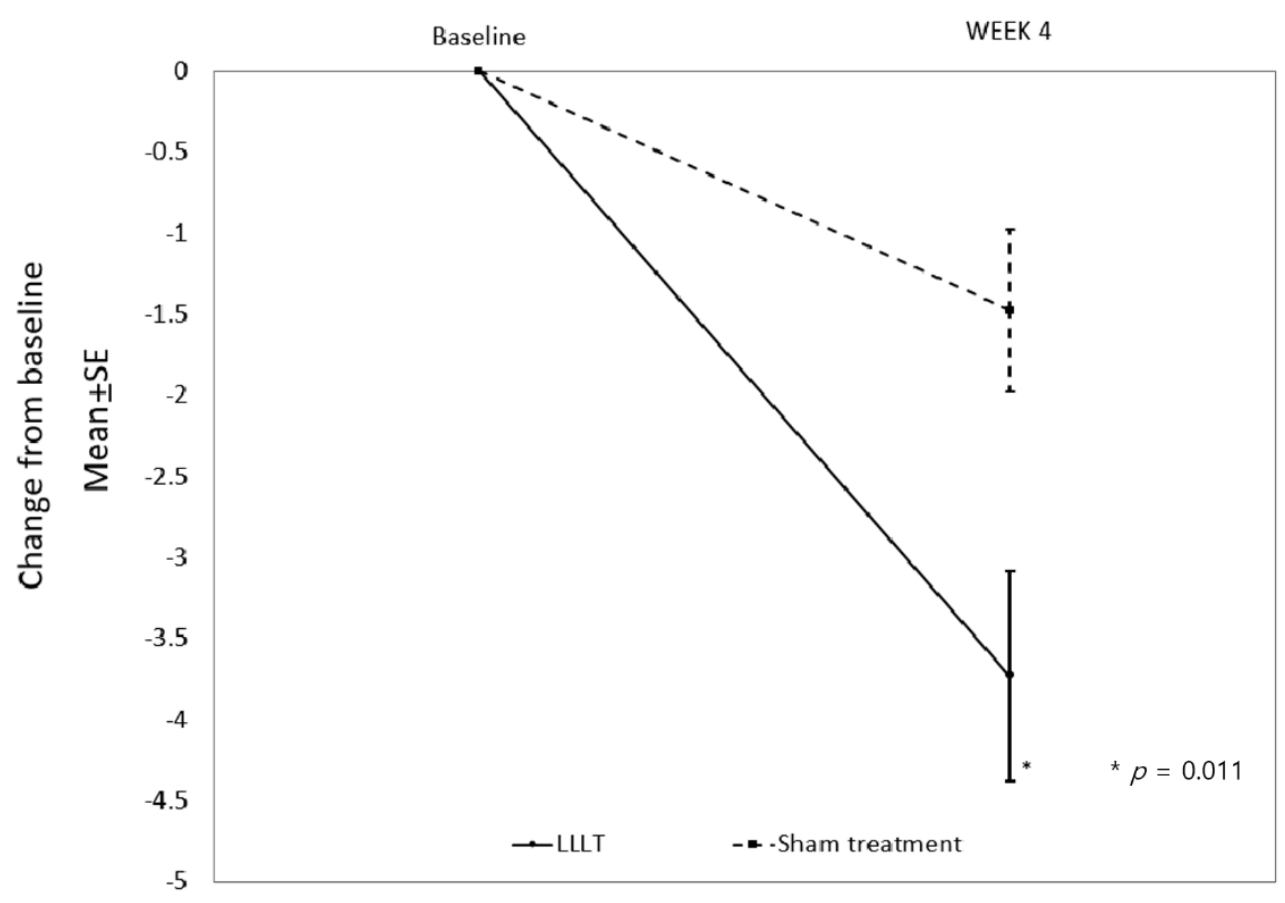

Figure 3. Least square means (LSMeans) in the TNSS from baseline to the end of treatment.

\subsection{Secondary Outcome}

In the LLLT group, the RQLQ score was $60.50 \pm 27.43$ at the baseline and decreased to $39.05 \pm 27.01$ at the end of the treatment period $(p<0.001)$. In the sham treatment group, the RQLQ score was $57.63 \pm 25.07$ at baseline and decreased to $41.37 \pm 26.92$ $(p<0.001)$. A greater reduction in RQLQ score was noted in the LLLT group without statistical significance $(p=0.383$, Table 5$)$.

Table 5. Treatment effects (RQLQ, mPP set).

\begin{tabular}{cccccc}
\hline Compliance & Group & Baseline & $\begin{array}{c}\text { End of } \\
\text { Treatment }\end{array}$ & Difference & $\begin{array}{c}\text { Comparison of } \\
\text { Difference } \\
\text { between } \\
\text { Groups: } \boldsymbol{p} \text {-Value }\end{array}$ \\
\hline \multirow{2}{*}{$\begin{array}{c}\text { (Modified } \\
\text { Per-Protocol) }\end{array}$} & LLLT $(n=22)$ & $60.50 \pm 27.43$ & $39.05 \pm 27.01$ & $-21.45 \pm 14.79$ & \multirow{2}{*}{0.383} \\
\cline { 2 - 5 } & Sham treatment $(n=19)$ & $57.63 \pm 25.07$ & $41.37 \pm 26.92$ & $-16.26 \pm 22.58$ & \\
\hline
\end{tabular}

Among the seven domains of the RQLQ, there were no significant differences in the reduction in following domains between groups: daily activities (difference $=0.60, p=0.596$ ), sleeping state (difference $=0.84, p=0.464$ ), eye symptoms (difference $=0.30, p=0.745$ ), non-nasal /eye symptoms (difference $=0.04, p=0.977$ ), practical problems (difference $=1.18$, $p=0.272$ ), and emotional function (difference $=0.001, p=1.000$ ). However, in the nasal symptoms domain, the mPP analysis showed that the RQLQ score was significantly reduced from 11.27 to 7.18 (difference $=4.09$ ) in the LLLT group, and 9.63 to 8.37 (difference $=1.26)$ in the sham treatment group $(p=0.036 ; 95 \%$ CI, $-5.464,-0.191$, Table 6$)$, suggesting that LLLT improves nasal symptoms (Figure 4, Table 6). 
Table 6. Treatment effects (RQLQ domains, mPP set).

\begin{tabular}{|c|c|c|c|c|c|}
\hline Domains & Group & Baseline & End of Treatment & Difference & $p$-Value \\
\hline \multirow{2}{*}{ Nasal symptoms } & $\operatorname{LLLT}(n=22)$ & $11.27 \pm 4.41$ & $7.18 \pm 4.24$ & $-4.09 \pm 3.65$ & \multirow{2}{*}{0.036} \\
\hline & Sham treatment $(n=19)$ & $9.63 \pm 3.35$ & $8.37 \pm 4.65$ & $-1.26 \pm 4.69$ & \\
\hline \multirow{2}{*}{ Daily activities } & $\operatorname{LLLT}(n=22)$ & $7.32 \pm 2.71$ & $4.45 \pm 2.94$ & $-2.86 \pm 2.78$ & \multirow{2}{*}{0.596} \\
\hline & Sham treatment $(n=19)$ & $7.74 \pm 3.36$ & $5.47 \pm 3.88$ & $-2.26 \pm 4.33$ & \\
\hline \multirow{2}{*}{ Sleeping state } & $\operatorname{LLLT}(n=22)$ & $7.09 \pm 4.47$ & $4.77 \pm 3.78$ & $-2.32 \pm 3.68$ & \multirow{2}{*}{0.464} \\
\hline & Sham treatment $(n=19)$ & $5.84 \pm 3.73$ & $4.37 \pm 3.68$ & $-1.47 \pm 3.60$ & \\
\hline \multirow{2}{*}{ Eye symptoms } & LLLT $(n=22)$ & $6.95 \pm 5.72$ & $4.36 \pm 4.48$ & $-2.59 \pm 2.81$ & \multirow{2}{*}{0.745} \\
\hline & Sham treatment $(n=19)$ & $6.89 \pm 5.30$ & $4.00 \pm 3.57$ & $-2.89 \pm 3.14$ & \\
\hline \multirow{2}{*}{$\begin{array}{l}\text { Non-nasal/eye } \\
\text { symptoms }\end{array}$} & $\operatorname{LLLT}(n=22)$ & $14.18 \pm 7.76$ & $9.77 \pm 7.37$ & $-4.41 \pm 4.15$ & \multirow{2}{*}{0.977} \\
\hline & Sham treatment $(n=19)$ & $13.32 \pm 6.38$ & $8.95 \pm 6.39$ & $-4.37 \pm 4.73$ & \\
\hline \multirow{2}{*}{ Practical problems } & LLLT $(n=22)$ & $7.82 \pm 3.25$ & $4.64 \pm 3.65$ & $-3.18 \pm 2.46$ & \multirow{2}{*}{0.272} \\
\hline & Sham treatment $(n=19)$ & $8.05 \pm 3.26$ & $6.05 \pm 4.14$ & $-2.00 \pm 3.99$ & \\
\hline \multirow{2}{*}{ Emotional function } & LLLT $(n=22)$ & $5.86 \pm 4.55$ & $3.86 \pm 3.72$ & $-2.00 \pm 2.88$ & \multirow{2}{*}{1.000} \\
\hline & Sham treatment $(n=19)$ & $6.16 \pm 4.30$ & $4.16 \pm 4.11$ & $-2.00 \pm 2.71$ & \\
\hline
\end{tabular}

Abbreviations: RQLQ, Rhinoconjunctivitis Quality of Life Questionnaire; LLLT, low-level laser therapy; mPP set, modified Per-Protocol set. The $p$-values were obtained from a chi-squared test and Wilcoxon's rank-sum test.

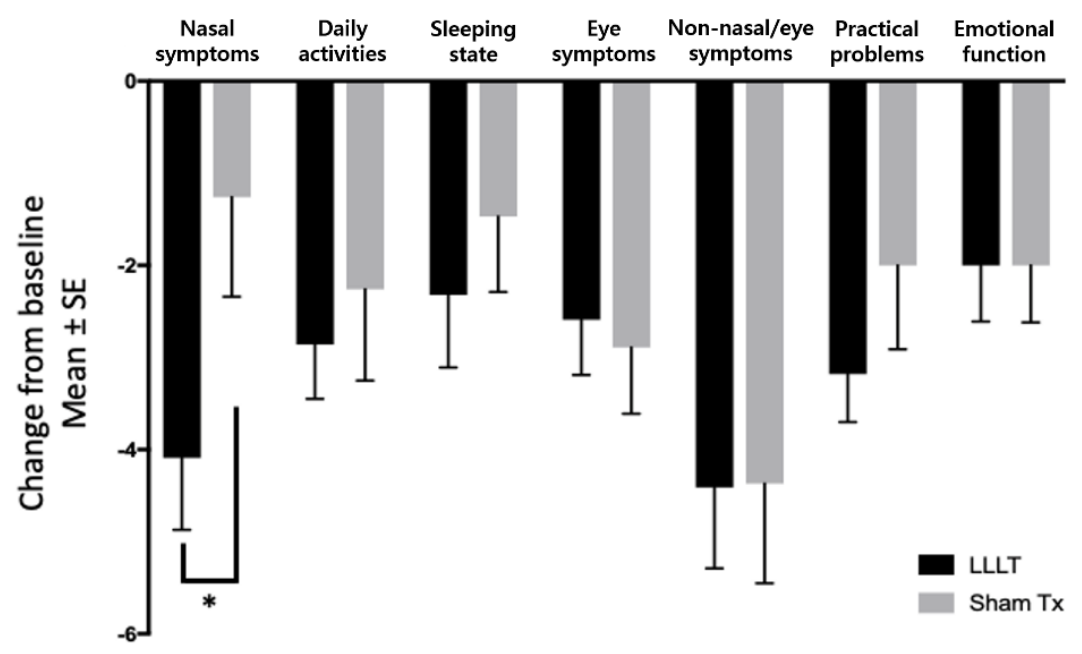

Figure 4. Seven domains of the RQLQ change from baseline. ${ }^{*} p<0.05$.

\subsection{Adverse Events}

Safety analysis was performed in the PP set. Some adverse events were reported by the participants during the treatment period (three in the LLLT group, five in the sham treatment group). In the LLLT group, all of the events were classified as definitely unrelated to the treatment. In the sham treatment group, $60.0 \%(n=3)$ of the events were classified as definitely unrelated to the treatment, and $40 \%(n=2)$ as possibly unrelated (Table 7$)$. There was no significant difference in the frequency of adverse events between the two groups $(p=0.476)$. No serious adverse events were reported or observed during the study. 
Table 7. Reported adverse events associated with LLLT and sham treatment participants.

\begin{tabular}{cc}
\hline Group & Adverse Events $(n)$ \\
\hline LLLT group & Definitely unrelated: idiopathic sudden hearing loss (1), injury of \\
& unrelated body part (1), pain of unrelated body part (1) \\
& Definitely unrelated: dyspepsia (1), pain of unrelated body part \\
Sham treatment group & $(1)$, incidental finding of unrelated body part (1) \\
& Possibly unrelated: acne (1), upper respiratory infection (1) \\
\hline
\end{tabular}

\section{Discussion}

This randomized, double-blind, placebo-controlled trial revealed that LLLT significantly suppressed the clinical symptoms of perennial AR at the end of the four-week treatment. The symptoms were improved to a greater extent in the LLLT treatment group. In addition, participants who received LLLT reported their quality of life regarding nasal symptoms to be much improved compared to those who received the sham treatment. In addition, there was no difference in the number of participants who reported adverse events, and there were no dropouts due to adverse events. Based on these findings, we recommend LLLT for the control of AR symptoms.

The mechanisms underlying LLLT have not been fully understood. However, possible mechanisms of action of LLLT in the treatment of AR have been studied previously $[10,13-18]$. The benefits of LLLT in AR may be primarily explained by anti-inflammatory mechanisms [21,22]. In animal models of acute pulmonary inflammation, LLLT relieved airway inflammation through the induction of IL-10 and reduction in the expression of macrophage inflammatory protein 2 and tumor necrosis factor (TNF) [23]. Koreck et al. reported that irradiation of the nasal mucosa resulted in a local reduction in IL-5, a cytokine that promotes the activation, maturation and prolonged survival of eosinophils, which are the main effector cells in AR [10]. Low-level lasers have been irradiated into the intranasal cavity of an ovalbumin-induced AR mouse model. Its inhibitory effect on immunoglobulin E and IL-4 production was histologically demonstrated via a reduced number of infiltrated inflammatory cells in the intranasal epithelium [18]. A review article concluded that LLLT reduces the concentration of signal molecules involved in the inflammatory response, and that LLLT can inhibit TNF- $\alpha$, cyclooxygenase-2, prostaglandin E2, and IL-1 $\beta$ [24]. All these effects of LLLT are desirable for improving symptoms among patients with AR.

Clinically, Csoma et al. showed that $308 \mathrm{~nm}$ of ultraviolet B irradiation significantly minimized AR symptoms, including nasal congestion, rhinorrhea, and sneezing, and improved the total nasal symptom scores and allergen-induced skin prick test results [25]. In a previous study, Garaczi et al. even insisted that intranasal irradiation with a combination of low-dose ultraviolet $B$ and visible light was significantly better than fexofenadine hydrochloride for the treatment of seasonal AR [26]. In a pilot study conducted in Korea that included 42 patients with perennial AR, nasal LLLT with a $650 \mathrm{~nm}$ laser significantly reduced the symptoms of AR [12]. In previous studies, the participants were patients with moderate to severe AR. However, the participants of this study had mild to moderate AR with a TNSS $\leq 9$. In addition, in contrast to the aforementioned studies, the participants in this study stopped any medication prior to enrollment in the study and were not allowed to use any AR symptom relievers throughout the course of treatment.

Dryness of the nasal mucosa, erythema, skin pain, pruritus, and pigmentation are potential LLLT treatment-specific adverse events mentioned in previous studies $[10,13,15]$. Dryness was not severe, did not increase the likelihood of nasal bleeding, and was easily controlled with emollients 2-3 times a day. Furthermore, it has been reported that dryness does not last after the end of treatment. In this study, none of the patients experienced dryness. Additionally, thermal effects may be a concern; however, low-level laser is a form of light emission with a power output of less than $500 \mathrm{~mW}$, and is considered as a type of nonthermal irradiation to living tissue. Another concern was that ultraviolet light, which can be mutagenic and carcinogenic, may cause DNA damage $[27,28]$. However, several studies have shown that nasal mucosae exposed to ultraviolet light have the capacity 
to repair DNA damage, which suggests that the multistep process of carcinogenesis has not been triggered, and no residual damage was seen in human subjects exposed to multiple ultraviolet B treatments $[15,29,30]$. Mitchell et al. showed that the nasal mucosa was capable of efficient repair of ultraviolet-induced DNA damage and suggested that ultraviolet phototherapy can be used in the treatment of AR [29]. The visible and infrared light used in this study have been shown to be safe. In addition, lasers with wavelengths of $700-1400 \mathrm{~nm}$ are classified in the "retinal hazard region"; however, in this study, we only used $3 \mathrm{~mW}$ and $20 \mathrm{~mW}$ lasers, which are not hazardous to the eye.

This study had some limitations. Poor compliance in the use of the device for the trial $(<50 \%)$ was observed in both groups (LLLT group, $n=10(31.3 \%)$; and sham treatment group, $n=14(42.4 \%))$. In the clinical trial of the newly developed device, it was important to evaluate the effect when compliant to some extent. Therefore, participants with poor compliance were judged as having non-sufficient exposure to study treatment and excluded, and it was thought that this was the basis for the use of mPP analysis to evaluate efficacy in more optimal conditions. In addition, there was no difference in compliance between the two groups of mPP. In the final mPP analysis, the sample size estimated was not achieved, and the verification power was statistically lowered. The reasons for the poor compliance could be speculated as follows; firstly, the AR symptoms among the participants were mild to moderate, which might have contributed to the poor compliance. Secondly, the $20 \mathrm{~min}$ usage time twice per day with the device in the nose could bother the participants to make them use it less. With the efforts to improve the compliance of device, more verifiable study and statistical analysis should be conducted later. The second limitation was the short follow-up period. The structural changes and biochemical activity induced by the procedure would reduce inflammation in the tissue but resolve later. Therefore, additional studies with longer follow-up periods are needed to evaluate the duration of the effects of LLLT. In addition, although LLLT appears to be safe and well tolerated, it was unclear if long-term low-level laser irradiation or thermal exposure of the nasal mucosa could result in DNA damage or irreversible changes. Long-term follow-up studies should be conducted in the future. Thirdly, treatment was only administered for four weeks, and the optimal duration for treatment with LLLT is unknown. Thus, it is possible that shorter durations could have a similar effect, and that longer use of the treatment or repeated treatments may enhance the outcome. Further studies are required to verify this. The fourth limitation was TNSS changes from the baseline at the final assessment was used in this study. The FDA recommended the change from baseline in the TNSS averaged over the entire treatment period as the primary endpoint. However, several other clinical trials also used TNSS changes, like in this study [31,32]. Despite these limitations, the present study was conducted according to a rigorous protocol, with a relatively large sample in a double-blind randomized and sham-controlled trial. In this regard, the results of this study are meaningful and encouraging.

\section{Conclusions}

We have demonstrated a significant reduction in the symptoms of perennial AR and an improved quality of life regarding nasal symptoms in patients treated with LLLT compared to those treated with the sham treatment. The results suggest that LLLT might be an effective and safe treatment for controlling the symptoms of AR.

Author Contributions: Conceptualization, P.S.C. and J.-H.M.; methodology, Y.-J.C.; formal analysis, Y.-J.C. and P.S.C.; investigation, H.J.J. and Y.-S.C.; data curation, H.J.J.; writing-original draft preparation, H.J.J. and Y.-S.C.; writing-review and editing, Y.-J.C., P.S.C. and J.-H.M.; visualization, H.J.J.; supervision, J.-H.M. All authors have read and agreed to the published version of the manuscript.

Funding: This research was supported by grants from the Ministry of Education (NRF-2020R1A6A 1A03043283), the Ministry of Science and Technology (NRF-2018K1A4A3A02060572), and the Ministry of Health and Welfare (HI15C1424, HI20C2088), Korea. 
Institutional Review Board Statement: The study was conducted according to the guidelines of the Declaration of Helsinki, approved by the Institutional Review Board of Dankook University Hospital (IRB no. 2016-09-011) and registered in the clinical research information system of South Korea (CRIS Registration Number KCT0003291).

Informed Consent Statement: Informed consent was obtained from all subjects involved in the study.

Data Availability Statement: Not applicable.

Conflicts of Interest: The authors declare no conflict of interest.

\section{References}

1. Bousquet, J.; Schünemann, H.J.; Togias, A.; Bachert, C.; Erhola, M.; Hellings, P.W.; Klimek, L.; Pfaar, O.; Wallace, D.; Ansotegui, I.; et al. Next-generation Allergic Rhinitis and Its Impact on Asthma (ARIA) guidelines for allergic rhinitis based on Grading of Recommendations Assessment, Development and Evaluation (GRADE) and real-world evidence. J. Allergy Clin. Immunol. 2020, 145, 70-80. [CrossRef]

2. Meltzer, E.O.; Blaiss, M.S.; Naclerio, R.M.; Stoloff, S.W.; Derebery, M.J.; Nelson, H.S.; Boyle, J.M.; Wingertzahn, M.A. Burden of allergic rhinitis: Allergies in America, Latin America, and Asia-Pacific adult surveys. Allergy Asthma Proc. 2012, 33, $113-141$. [CrossRef]

3. Caballer, B.D.L.H.; Rodríguez, M.; Fraj, J.; Cerecedo, I.; Antolín-Amérigo, D.; Colas, C. Allergic Rhinitis and Its Impact on Work Productivity in Primary Care Practice and a Comparison with Other Common Diseases: The Cross-sectional Study to Evaluate Work Productivity in Allergic Rhinitis Compared with Other Common Diseases (CAPRI) Study. Am. J. Rhinol. Allergy 2012, 26, 390-394. [CrossRef]

4. Bousquet, J.; Anto, J.M.; Bachert, C.; Baiardini, I.; Bosnic-Anticevich, S.; Walter Canonica, G.; Melén, E.; Palomares, O.; Scadding, G.K.; Togias, A.; et al. Allergic rhinitis. Nat. Rev. Dis. Primers 2020, 6, 95. [CrossRef]

5. Meltzer, E.O.; Nathan, R.; Derebery, J.; Stang, P.E.; Campbell, U.B.; Yeh, W.-S.; Corrao, M.; Stanford, R. Sleep, quality of life, and productivity impact of nasal symptoms in the United States: Findings from the Burden of Rhinitis in America survey. Allergy Asthma Proc. 2009, 30, 244-254. [CrossRef]

6. Felix, M.; Paz, C.V.; Mata, V.L.; Vanegas, E.; Larenas-Linnemann, D.; Rosario, N.A.; Letort, J.; Cherrez-Ojeda, I. Perceptions and Management of Allergic Rhinitis Among Ecuadorian Otorhinolaryngologists: A Survey-Based Study. J. Multidiscip. Healthc. 2020, 13, 1975-1981. [CrossRef] [PubMed]

7. Bernstein, J.A. Allergic and mixed rhinitis: Epidemiology and natural history. Allergy Asthma Proc. 2010, 31, 365-369. [CrossRef] [PubMed]

8. Nathan, R.A. Management of Patients with Allergic Rhinitis and Asthma: Literature Review. South. Med. J. 2009, 102, 935-941. [CrossRef] [PubMed]

9. Csoma, Z.; Koreck, A.; Ignacz, F.; Bor, Z.; Szabo, G.; Bodai, L.; Dobozy, A.; Kemeny, L. PUVA treatment of the nasal cavity improves the clinical symptoms of allergic rhinitis and inhibits the immediate-type hypersensitivity reaction in the skin. $J$. Photochem. Photobiol. B Biol. 2006, 83, 21-26. [CrossRef] [PubMed]

10. Koreck, A.I.; Csoma, Z.; Bodai, L.; Ignacz, F.; Kenderessy, A.S.; Kadocsa, E.; Szabo, G.; Bor, Z.; Erdei, A.; Szony, B. Rhinophototherapy: A new therapeutic tool for the management of allergic rhinitis. J. Allergy Clin. Immunol. 2005, 115, 541-547. [CrossRef]

11. Hu, K.-H.; Li, W.-T. Clinical Effects of Far-Infrared Therapy in Patients with Allergic Rhinitis. In Proceedings of the 29th Annual International Conference of the IEEE Engineering in Medicine and Biology Society, Lyon, France, 23-26 August 2007; Volume 2007, pp. 1479-1482.

12. Lee, H.M.; Park, M.S.; Park, I.H.; Lee, S.H.; Lee, S.K.; Kim, K.S.; Choi, H. A comparative pilot study of symptom improvement before and after phototherapy in Korean patients with perennial allergic rhinitis. Photochem. Photobiol. 2013, 89, 751-757. [CrossRef]

13. Cingi, C.; Cakli, H.; Yaz, A.; Songu, M.; Bal, C. Phototherapy for allergic rhinitis: A prospective, randomized, single-blind, placebo-controlled study. Ther. Adv. Respir. Dis. 2010, 4, 209-213. [CrossRef]

14. Kemény, L.; Koreck, A. Ultraviolet light phototherapy for allergic rhinitis. J. Photochem. Photobiol. B Biol. 2007, 87, 58-65. [CrossRef]

15. Koreck, A.; Szechenyi, A.; Morocz, M.; Cimpean, A.; Bella, Z.; Garaczi, E.; Raica, M.; Olariu, T.; Rasko, I.; Kemeny, L. Effects of intranasal phototherapy on nasal mucosa in patients with allergic rhinitis. J. Photochem. Photobiol. B Biol. 2007, 89, 163-169. [CrossRef]

16. Aimbire, F.; Albertini, R.; Pacheco, M.T.; Castro-Faria-Neto, H.C.; Leonardo, P.S.; Iversen, V.V.; Lopes-Martins, R.A.; Bjordal, J.M. Low-level laser therapy induces dose-dependent reduction of TNFalpha levels in acute inflammation. Photomed. Laser Surg. 2006, 24, 33-37. [CrossRef] [PubMed]

17. De Oliveira, R.G.; Aarestrup, F.M.; Miranda, C.; Vieira, B.J.; Ferreira, A.P.; Andrade, L.C. Low-Level Laser Therapy Reduces Delayed Hypersensitivity Reaction to Ovalbumin in Balb/C Mice. Photomed. Laser Surg. 2010, 28, 773-777. [CrossRef] [PubMed] 
18. Choi, B.; Chang, M.S.; Kim, H.Y.; Park, J.-W.; Ryu, B.; Kim, J. Effects of Low Level Laser Therapy on Ovalbumin-Induced Mouse Model of Allergic Rhinitis. Evidence-Based Complement. Altern. Med. 2013, 2013, 1-8. [CrossRef] [PubMed]

19. Hampel, F.C.; Ratner, P.H.; Van Bavel, J.; Amar, N.; Daftary, P.; Wheeler, W.; Sacks, H. Double-blind, placebo-controlled study of azelastine and fluticasone in a single nasal spray delivery device. Ann. Allergy Asthma Immunol. 2010, 105, 168-173. [CrossRef]

20. Juniper, E.F.; Thompson, A.K.; Ferrie, P.J.; Roberts, J.N. Validation of the standardized version of the Rhinoconjunctivitis Quality of Life Questionnaire. J. Allergy Clin. Immunol. 1999, 104, 364-369. [CrossRef]

21. Krespi, Y.P.; Kizhner, V. Phototherapy for chronic rhinosinusitis. Lasers Surg. Med. 2011, 43, 187-191. [CrossRef]

22. Bjordal, J.M.; Lopes-Martins, R.Á.B.; Joensen, J.; Iversen, V.V. The anti-inflammatory mechanism of low level laser therapy and its relevance for clinical use in physiotherapy. Phys. Ther. Rev. 2010, 15, 286-293. [CrossRef]

23. De Lima, F.M.; Villaverde, A.B.; Albertini, R.; Correa, J.C.; Carvalho, R.L.P.; Munin, E.; Araújo, T.; Silva, J.A.; Aimbire, F. Dual Effect of low-level laser therapy (LLLT) on the acute lung inflammation induced by intestinal ischemia and reperfusion: Action on anti- and pro-inflammatory cytokines. Lasers Surg. Med. 2011, 43, 410-420. [CrossRef]

24. Gao, X.; Xing, D. Molecular mechanisms of cell proliferation induced by low power laser irradiation. J. Biomed. Sci. 2009, 16, 4 . [CrossRef] [PubMed]

25. Csoma, Z.; Ignacz, F.; Bor, Z.; Szabo, G.; Bodai, L.; Dobozy, A.; Kemény, L. Intranasal irradiation with the xenon chloride ultraviolet B laser improves allergic rhinitis. J. Photochem. Photobiol. B Biol. 2004, 75, 137-144. [CrossRef]

26. Garaczi, E.; Koreck, A.; Boros-Gyevi, M.; Bella, Z.; Csoma, Z.; Kemény, L. Intranasal Phototherapy Is More Effective Than Fexofenadine Hydrochloride in the Treatment of Seasonal Allergic Rhinitis: Results of a Pilot Study. Photochem. Photobiol. 2011, 87, 474-477. [CrossRef]

27. Cadet, J.; Sage, E.; Douki, T. Ultraviolet radiation-mediated damage to cellular DNA. Mutat. Res. Mol. Mech. Mutagen. 2005, 571, 3-17. [CrossRef]

28. Kuluncsics, Z.; Perdiz, D.; Brulay, E.; Muel, B.; Sage, E. Wavelength dependence of ultraviolet-induced DNA damage distri-bution: Involvement of direct or indirect mechanisms and possible artefacts. J. Photochem. Photobiol. B 1999, 49, 71-80. [CrossRef]

29. Mitchell, D.; Paniker, L.; Sánchez, G.; Bella, Z.; Garaczi, E.; Szell, M.; Hamid, Q.; Kemény, L.; Koreck, A. Molecular response of nasal mucosa to therapeutic exposure to broad-band ultraviolet radiation. J. Cell. Mol. Med. 2008, 14, 313-322. [CrossRef] [PubMed]

30. Leong, S.C. Rhinophototherapy: Gimmick or an emerging treatment option for allergic rhinitis? Rhinol. J. 2011, 49, 499-506.

31. Okubo, K.; Ichimura, M.; Koyama, T.; Susuta, Y.; Izaki, H. Double-blind placebo-controlled study of bepotastine besilate in pediatric patients with perennial allergic rhinitis. Expert Opin. Pharmacother. 2015, 16, 2395-2408. [CrossRef] [PubMed]

32. Mo, J.H.; Jeong, J.H.; Kim, T.H.; Kim, S.T.; Kim, S.W.; Lee, K.H.; Hong, S.N.; Kim, H.Y.; Kim, D.W.; Kim, D.Y.; et al. Double-Blind Placebo-Controlled Trial of Bepotastine Salicylate in Patients With Allergic Rhinitis. Laryngoscope 2021, 131, E702-E709. [CrossRef] [PubMed] 